\title{
Emergence of High Drug Resistant Bacterial Isolates in Al-Kharj
}

\author{
Nehad Jaser Ahmed ${ }^{1,2,}$, Abdul Haseeb ${ }^{3}$, Salwa Mahmoud ${ }^{4}$, Amer Hayat Khan $^{2}$ \\ 'Department of Clinical Pharmacy, Pharmacy College, Prince Sattam Bin Abdulaziz University, AI-Kharj, SAUDI ARABIA. \\ ${ }^{2}$ Discipline of Clinical Pharmacy, School of Pharmaceutical Sciences, Universiti Sains Malaysia, 11800, Penang, MALAYSIA. \\ ${ }^{3}$ Clinical Pharmacy Department, College of Pharmacy, Umm Al-Qura University, SAUDI ARABIA. \\ ${ }^{4}$ Department of pathology, Al-Kharj Military Industrial Corporation Hospital, Al-Kharj, SAUDI ARABIA.
}

\begin{abstract}
Background: Continuous utilization of antimicrobial drugs in treating infections has led to the development of resistance among various strains of bugs. The aim of this study was to determine the prevalence of antibiotic-resistant bacteria in a military hospital in Al-Kharj. Methods: This study is a retrospective study that include collecting the results of bacterial cultures. After collecting the data, an antibiogram was prepared and after that the resistance rates for each bacterium were calculated by subtraction of $100 \%$ from the susceptibility rate for each result (Resistance rate $=100 \%$ - Susceptibility rate). Results: Acinetobacter baumannii was resistant to most tested antibiotics and Klebsiella pneumonia was resistant to several antibiotics in 2018 and 2019. In the present study, there were a high resistance rate of Klebsiella pneumonia and Acinetobacter baumannii and there were 2 main threats which include Carbapenem-resistant Acinetobacter and Ciprofloxacin-resistant Salmonella. Conclusion: It is recommended to
\end{abstract}

increase the awareness of the health care professionals regarding the appropriate prescribing of antibiotics based on antimicrobial resistance rate and to increase the awareness of the public about the wise use of antibiotics.

Key words: Antibiotics, Bacteria, Bacterial Isolates, Multidrug Resistant, Resistance.

\section{Correspondence}

Nehad Jaser Ahmed,

Department of Clinical Pharmacy, College of Pharmacy, Prince Sattam Bin Abdulaziz University, Al-Kharj, SAUDI ARABIA

Email id: n.ahmed@psau.edu.sa

DOI: 10.5530/jyp.2021.13.94

\section{INTRODUCTION}

Antibiotics are medications used to treat several infections. The era from 1930s to 1960s was considered the golden era of antibiotics which gave rise to numerous antibiotics. ${ }^{1}$ This era ended because the researchers were unable to keep up with the pace of antibiotic discovery in the face of emerging resistant bugs. The predisposing factors that are associated with the emergence of antibiotic resistance include the failure to discover or develop novel antibiotics and the inappropriate use of antibiotics. ${ }^{2}$ The development of antimicrobial resistance was observed shortly after the introduction of novel antimicrobial agents. ${ }^{3}$ Therefore, continuous utilization of antimicrobial drugs in treating infections has led to the development of resistance among numerous strains of bugs. Multidrug resistance is defined as the resistance or insensitivity of a microbe to antimicrobial medicine despite the sensitivity to it previously. ${ }^{4,5}$

Multidrug resistant bacteria are one of the main threats to public health. ${ }^{6-8}$ Typically, Multidrug resistant microbes are associated with nosocomial infections. However, some of these bacteria have become moderately prevalent causes of community-acquired infections. ${ }^{6}$ Multidrug resistant bacteria result in numerous negative consequences, the outcomes in patients infected with multidrug resistant bacteria tend to be worse than the outcomes in patients infected with more susceptible bacteria. ${ }^{9,10}$ Studies from World Health Organization report have shown very high rates of resistance in several bacteria such as Klebsiella pneumoniae against carbapenems and cephalosporin, Escherichia coli against antibiotics as fluoroquinolones and cephalosporin, Streptococcus pneumoniae against penicillin, Staphylococcus aureus against methicillin, Shigella species against fluoroquinolones, Nontyphoidal Salmonella against fluoroquinolones, Neisseria gonorrhoeae against cephalosporin and Mycobacterium tuberculosis against fluoroquinolone, rifampicin and isoniazid. ${ }^{11,12}$ An international panel of experts stated that the bacterial resistance is classified as multidrug-resistant if the bacteria acquired non-susceptibility to at least one antibiotic in three or more antibiotic classes. ${ }^{13}$

It is important to know the antibiotic resistance rate in clinical isolates to give the appropriate antibiotic treatment. Additionally, knowing the resistance rate could help health planners to develop strategies to prevent the spread of antibiotic resistance. There is a lack of studies about antibiotic-resistant bacteria in Al-Kharj. Therefore, the aim of this study was to determine the prevalence of antibiotic-resistant bacteria in a military hospital in Al-Kharj.

\section{MATERIALS AND METHODS}

\section{Setting}

The study was conducted at the Military Industrial Corporation Hospital in Al-Kharj. This hospital is initiated in 1979 in Alkharj that is located near Riyadh city, the capital of Saudi Arabia. The hospital includes only 60 beds, so it is considered a small hospital (with less than 100 beds).

\section{Inclusion/exclusion criteria}

The results of all bacterial cultures in 2018 and 2019 were included. Bacterial cultures before 2018 or after 2019 were excluded and the results of fungal cultures were also excluded.

\section{Data collection and analysis}

The data were collected from the bacterial cultures' results that were prepared by the help of Microbiology department in the hospital. The data was represented in 4 tables: the gram-negative bacterial resistance rates in 2018, the gram-positive bacterial resistance rates in 2018, the gram-negative bacterial resistance rates in 2019 and the gram 
positive bacterial resistance rates in 2019. After collecting the data, an antibiogram was prepared and after that the resistance rates for each bacterium were calculated by subtraction of $100 \%$ from the susceptibility rate for each result (Resistance rate $=100 \%$ - Susceptibility rate). If the bacteria are resistant to at least one antibiotic in three or more antibiotic classes, these bacteria are classified as multidrug-resistant bacteria.

\section{Ethical approval}

The study was approved by the IRB Ethical Committee of the hospital No: 4101728 . The study excluded any patient' identifiers to protect the patients' privacy.

\section{RESULTS}

\section{Total number of bacterial isolates in 2018 and 2019}

In 2018 and 2019, there were a total of 1735 bacterial isolates, the most prevalent bacteria were Escherichia coli bacteria (28.41\%), Staphylococcus aureus (22.54\%), Pseudomonas aeruginosa (11.64 \%), Klebsiella pneumonia (11.47\%) and group B Streptococcus (5.82\%). These 5 bacteria form 79.88 $\%$ of the total bacteria isolates in the 2 years.

\section{Number of bacterial isolates in 2018}

In 2018, there were 743 bacterial isolates. The most common organism was Escherichia coli (32.84\%) followed by Pseudomonas aeruginosa (14.14\%), Staphylococcus aureus (12.52\%) and Klebsiella pneumonia (10.50\%). Table 1 shows the total number of bacterial isolates in 2018.

Table 1: Total number of bacterial isolates in 2018.

\begin{tabular}{|c|c|c|}
\hline Bacteria & Number & Percentage \\
\hline Escherichia coli & 244 & 32.84 \\
\hline Pseudomonas aeruginosa & 105 & 14.14 \\
\hline Staphylococcus aureus & 93 & 12.52 \\
\hline Klebsiella pneumonia & 78 & 10.50 \\
\hline Acinetobacter baumannii & 51 & 6.87 \\
\hline Coagulase-ve Staphylococcus & 33 & 4.44 \\
\hline Group B Streptococcus & 30 & 4.04 \\
\hline Enterobacter cloacae & 20 & 2.69 \\
\hline Proteus mirabillis & 17 & 2.29 \\
\hline Enterococcus faecalis & 17 & 2.29 \\
\hline Salmonella & 11 & 1.48 \\
\hline Serratia marcescens & 10 & 1.35 \\
\hline Group A Streptococcus & 7 & 0.94 \\
\hline Enterobacter aerogenes & 5 & 0.67 \\
\hline Brucella & 5 & 0.67 \\
\hline Haemophillus Spp & 4 & 0.54 \\
\hline Providencia stuartii & 4 & 0.54 \\
\hline Morganella morganii & 3 & 0.40 \\
\hline Citrobacter Koseri & 3 & 0.40 \\
\hline Citrobacter youngea & 1 & 0.13 \\
\hline Pseudomonas fluorescens & 1 & 0.13 \\
\hline Citrobacter freundii & 1 & 0.13 \\
\hline Total & 743 & 100.00 \\
\hline
\end{tabular}

\section{Number of bacterial isolates in 2019}

In 2019, there were 992 bacterial isolates. The most common organism was Staphylococcus aureus (30.04\%) followed by Escherichia coli (25.10\%), Klebsiella pneumonia (12.20\%) and Pseudomonas aeruginosa (9.78\%). Table 2 shows the total number of bacterial isolates in 2019 .

\section{The resistance rate of gram negative and gram-positive bacteria in 2018}

The resistance rate for most of gram-negative bacteria in 2018 was generally low. Only there was a high resistance rate for Acinetobacter baumannii that was resistant to most tested antibiotics. Table 3 shows the gram-negative bacterial resistance rates in 2018. Regarding gram positive bacteria, the resistance rate generally was low and there were no multidrug resistant bacteria. The gram-positive bacterial resistance rates in 2018 are shown in Table 4.

\section{The resistance rate of gram negative and gram-positive bacteria in 2019}

The resistance rate for most of gram-negative bacteria in 2019 was generally low. Only there were high resistance rate for Acinetobacter baumannii and Klebsiella pneumonia. Acinetobacter baumannii was resistant to most tested antibiotics and Klebsiella pneumonia was resistant to several antibiotics. Table 5 shows the gram-negative bacterial resistance rates in 2019. Regarding gram positive bacteria, the resistance rate generally was low. Only Coagulase -ve Staphylococcus bacteria were resistant to 3 antibiotics, erythromycin, oxacillin, and clindamycin. The gram-positive bacterial resistance rates in 2019 are shown in Table 6.

\section{DISCUSSION}

In 2018 and 2019, the most prevalent bacteria were Escherichia coli (28.41\%), Staphylococcus aureus (22.54\%), Pseudomonas aeruginosa (11.64\%), Klebsiella pneumonia (11.47\%) and Group B Streptococcus $(5.82 \%)$. These 5 bacteria form approximately $80 \%$ of the total bacteria isolates. Likewise, Bono et al. reported that in patients with renal diseases the most frequently isolated bacterial pathogens were Escherichia coli and Staphylococcus aureus followed by Enterococcus faecalis, Pseudomonas

Table 2: Total number of bacterial isolates in 2019.

\begin{tabular}{ccc}
\hline Bacteria & Number & Percentage \\
\hline Staphylococcus aureus & 298 & 30.04 \\
Escherichia coli & 249 & 25.10 \\
Klebsiella pneumonia & 121 & 12.20 \\
Pseudomonas aeruginosa & 97 & 9.78 \\
Group B Streptococcus & 71 & 7.16 \\
Coagulase -ve Staphylococcus & 32 & 3.23 \\
Proteus mirabillis & 28 & 2.82 \\
Enterococcus faecalis & 21 & 2.12 \\
Acinetobacter baumannii & 19 & 1.91 \\
Enterobacter cloacae & 15 & 1.51 \\
Salmonella & 12 & 1.21 \\
Serratia marcescens & 10 & 1.01 \\
Group A Streptococcus & 9 & 0.91 \\
Enterobacter aerogenes & 5 & 0.50 \\
Providencia stuartii & 5 & 0.50 \\
Total & $\mathbf{1 0 0 . 0 0}$ \\
\hline
\end{tabular}


Table 3: The gram-negative bacterial resistance rates in 2018.

\begin{tabular}{|c|c|c|c|c|c|c|c|c|c|c|c|c|c|c|c|c|c|c|c|c|c|}
\hline Antibioti & \# & 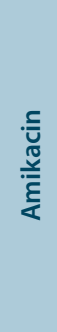 & 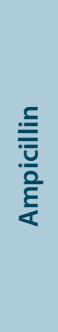 & 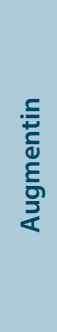 & 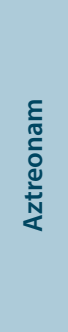 & 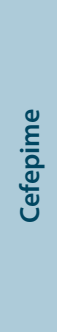 & 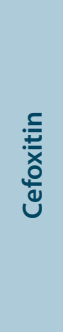 & 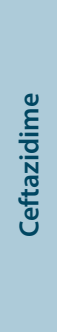 & 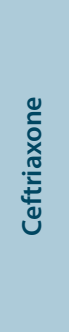 & 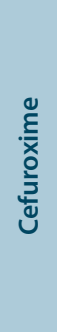 & 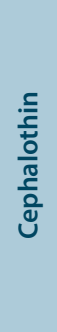 & 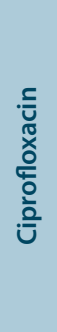 & 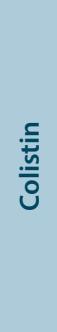 & 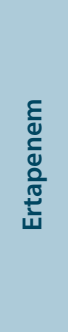 & 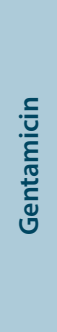 & 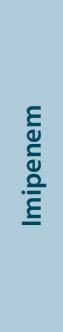 & 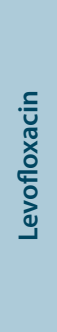 & 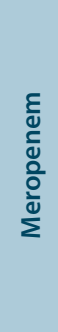 & 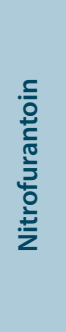 & 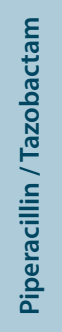 & 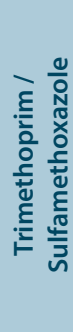 \\
\hline Escherichia coli & 244 & 0 & 60 & 37 & 30 & 28 & 7 & 28 & 27 & 29 & 82 & 25 & NA & 12 & 18 & 7 & 25 & 5 & 3 & 4 & 53 \\
\hline Enterobacter aerogenes & 5 & 0 & NA & NA & 20 & 20 & NA & 0 & 20 & NA & NA & 0 & NA & 0 & 20 & 0 & 0 & 0 & NA & 0 & 0 \\
\hline Enterobacter cloacae & 20 & 0 & NA & NA & 20 & 2 & NA & 0 & 20 & NA & NA & 0 & NA & 2 & 2 & 0 & 0 & 0 & NA & 20 & 0 \\
\hline Haemophillus Spp & 4 & $\mathrm{NA}$ & 0 & 0 & NA & NA & NA & NA & 25 & 25 & NA & 0 & NA & NA & NA & NA & NA & NA & NA & NA & 75 \\
\hline Klebsiella pneumonia & 78 & 5 & NA & 30 & 32 & 35 & 20 & 30 & 35 & 28 & 40 & 20 & NA & 25 & 15 & 18 & 16 & 18 & 55 & 25 & 30 \\
\hline Citrobacter freundii & 1 & 0 & NA & NA & 0 & 0 & NA & NA & NA & NA & NA & NA & NA & 0 & 0 & 0 & NA & 0 & 0 & 0 & 0 \\
\hline Citrobacter Koseri & 3 & 0 & NA & 0 & 0 & 0 & NA & 0 & 0 & 0 & 0 & 0 & NA & NA & 0 & 0 & 0 & 0 & NA & 0 & 0 \\
\hline Serratia marcescens & 10 & 15 & NA & NA & 15 & 15 & NA & 15 & 35 & NA & NA & 15 & NA & 35 & 15 & 35 & 15 & 35 & NA & 0 & 0 \\
\hline Proteus mirabillis & 17 & 10 & 48 & 27 & 14 & 14 & 0 & 14 & 14 & 29 & 43 & 100 & NA & 0 & 29 & NA & 29 & 0 & NA & 0 & 71 \\
\hline Providencia stuartii & 4 & 0 & NA & NA & 50 & 50 & 0 & 50 & 50 & 50 & NA & NA & NA & 50 & NA & 50 & NA & 50 & NA & 0 & 50 \\
\hline Morganella morganii & 3 & 0 & NA & NA & 0 & 0 & 0 & 0 & 0 & NA & NA & 34 & NA & 0 & 0 & 0 & 0 & 0 & NA & 0 & 0 \\
\hline Citrobacter youngea & 1 & 0 & NA & NA & 0 & 0 & NA & 0 & 0 & 0 & NA & 0 & NA & 0 & 0 & 0 & 0 & 0 & NA & 0 & 0 \\
\hline Pseudomonas fluorescens & 1 & 0 & NA & NA & 0 & 0 & NA & 0 & NA & 0 & NA & 0 & 0 & NA & 0 & 0 & 0 & 0 & NA & 100 & NA \\
\hline Salmonella & 11 & NA & 28 & NA & NA & NA & NA & NA & 0 & NA & NA & 70 & NA & NA & NA & NA & NA & NA & NA & NA & 34 \\
\hline Pseudomonas eruginosa & 105 & 0 & NA & NA & 15 & 17 & NA & 15 & NA & NA & NA & 21 & 10 & NA & 10 & 20 & 20 & 18 & NA & 10 & NA \\
\hline Acinetobacter baumannii & 51 & 45 & NA & NA & NA & 60 & NA & 60 & 70 & NA & NA & 60 & 1 & NA & 67 & 67 & 70 & 67 & NA & 67 & 20 \\
\hline Brucella & 5 & NA & NA & NA & NA & NA & NA & NA & NA & NA & NA & 0 & NA & NA & 0 & NA & NA & NA & NA & NA & 25 \\
\hline
\end{tabular}

Table 4: The gram-positive bacterial resistance rates in 2018.

\begin{tabular}{|c|c|c|c|c|c|c|c|c|c|c|c|c|c|c|c|c|c|c|c|c|c|c|}
\hline acteria & \# & 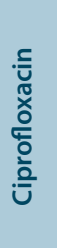 & 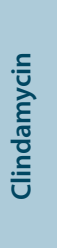 & 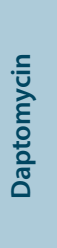 & 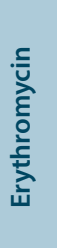 & 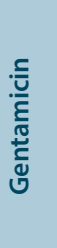 & 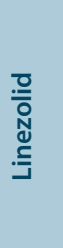 & 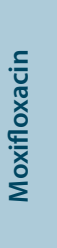 & 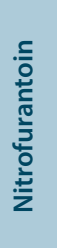 & 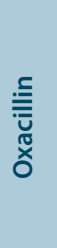 & 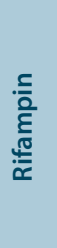 & 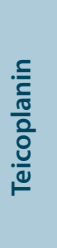 & 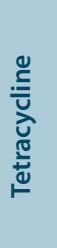 & 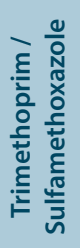 & 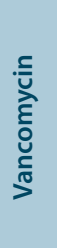 & 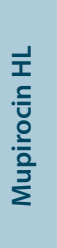 & 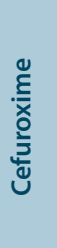 & 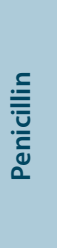 & 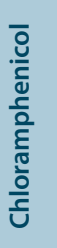 & 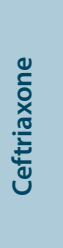 & 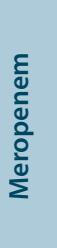 & 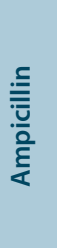 \\
\hline Staphylococcus aureus & 93 & 13 & 17 & 2 & 29 & 7 & 0 & 0 & 0 & 37 & 0 & 3 & 15 & 19 & 0 & 2 & NA & NA & NA & NA & NA & NA \\
\hline Coagulase -ve Staphylococcus & 33 & 70 & 30 & 20 & 40 & 30 & 2 & 0 & 0 & 60 & 10 & 10 & 15 & 40 & , & 2 & NA & NA & NA & NA & NA & NA \\
\hline Group A Streptococcus & 7 & NA & NA & NA & 0 & NA & NA & NA & NA & NA & NA & NA & NA & 0 & 0 & NA & 0 & 0 & 0 & NA & NA & NA \\
\hline Group B Streptococcus & 30 & NA & NA & NA & 40 & NA & NA & NA & NA & NA & NA & NA & 70 & 30 & 5 & NA & 0 & 5 & 15 & 5 & 10 & NA \\
\hline Enterococcus faecalis & 17 & 45 & NA & NA & NA & 45 & 0 & NA & NA & NA & NA & NA & NA & NA & 0 & NA & NA & NA & NA & NA & NA & 15 \\
\hline
\end{tabular}

aeruginosa, Staphylococcus epidermidis and Klebsiella pneumonia. ${ }^{14}$ Ahmed et al. reported that ESKAPE pathogens (Enterococcus faecium, Staphylococcus aureus, Klebsiella pneumoniae, Acinetobacter baumannii, Pseudomonas aeruginosa and Enterobacter spp.) cause nearly half of the infections that were caused by bacteria and the most common ESKAPE pathogens were Klebsiella pneumonia and Pseudomonas aeruginosa. ${ }^{15}$

The resistance rate for most of gram-negative bacteria in the present study in 2018 was generally low. Escherichia coli was highly resistant to cephalothin (82\%), ampicillin (60\%) and to trimethoprim / sulfamethoxazole (53\%). Klebsiella pneumonia was highly resistant to nitrofurantoin (55\%) but the number of Klebsiella pneumonia bacteria were low
(17 isolates). Proteus mirabillis bacteria was highly resistant to ciprofloxacin (100\%) and to trimethoprim / sulfamethoxazole (71\%). Salmonella was highly resistant to ciprofloxacin (70\%) but the number of Salmonella bacteria were low (11 isolates). In 2018, there was a high resistance rate for Acinetobacter baumannii which was resistant to most of the tested antibiotics. For example, the resistance rate of Acinetobacter baumannii was $70 \%$ to ceftriaxone and levofloxacin. The resistance rate was $67 \%$ to gentamicin, imipenem, meropenem and piperacillin / tazobactam. The resistance rate was $60 \%$ to cefepime, ceftazidime and ciprofloxacin.

The resistance rate for most of gram-negative bacteria in the present study in 2019 was generally low. Escherichia coli was highly resistant to 


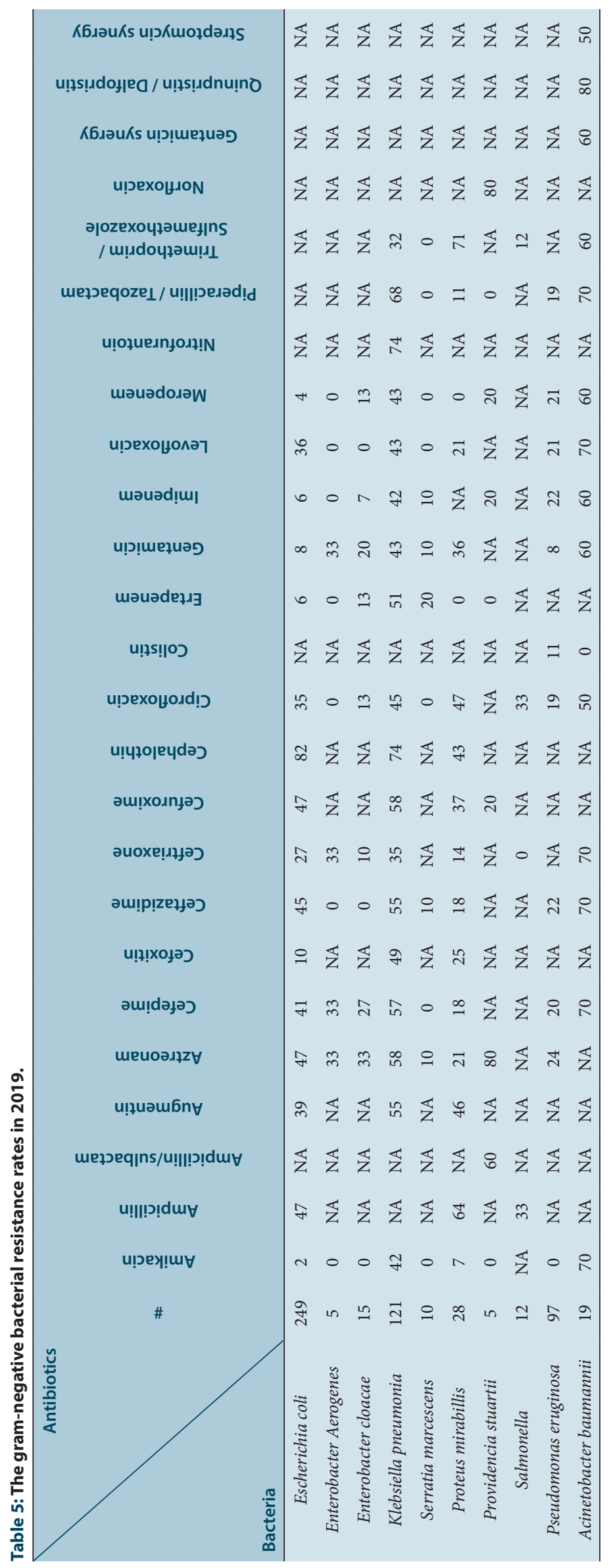

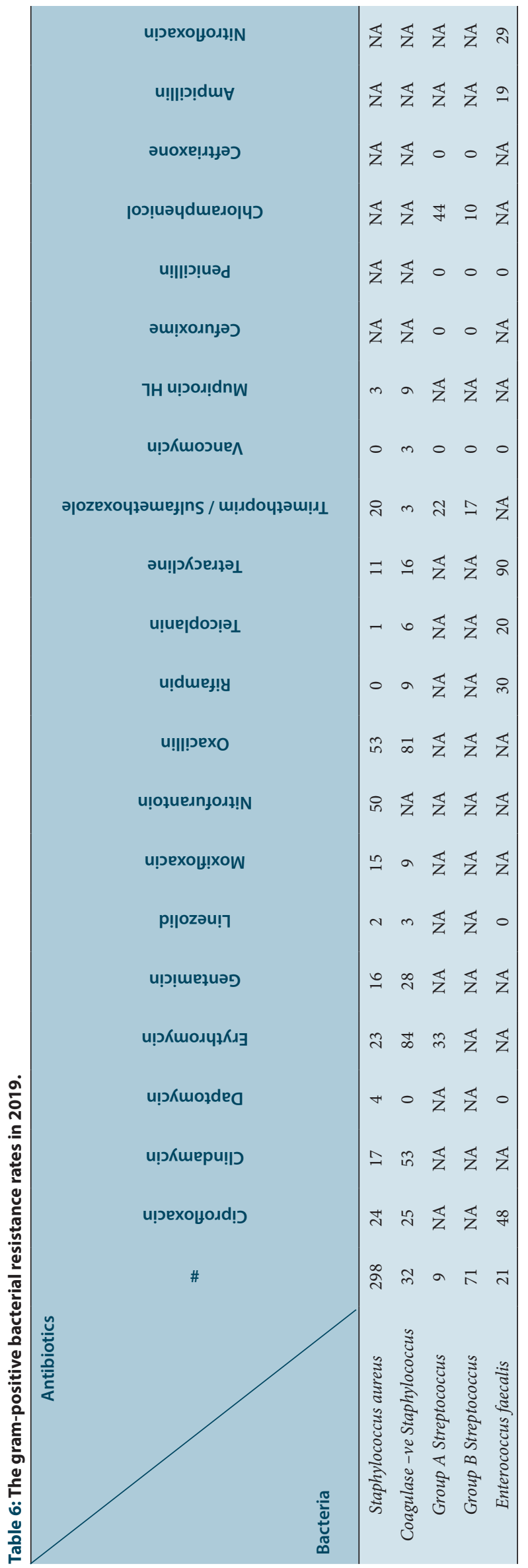


cephalothin (82\%). Klebsiella pneumonia was highly resistant to nitrofurantoin (74\%), cephalothin (74\%), piperacillin / tazobactam (68\%), aztreonam (58\%), cefuroxime (58\%), augmentin (55\%), ceftazidime (55\%) and ertapenem (51\%). Acinetobacter baumannii was highly resistant to quinupristin / dalfopristin (80\%). The resistance rate of Acinetobacter baumannii to amikacin, cefepime, ceftazidime, ceftriaxone, levofloxacin, piperacillin / tazobactam was $70 \%$. Moreover, the resistance rate of Acinetobacter baumannii was $60 \%$ to gentamicin, imipenem, meropenem, trimethoprim / sulfamethoxazole and gentamicin synergy. The resistance rate was $50 \%$ to ciprofloxacin and streptomycin synergy. Nevertheless, the resistance rate of Acinetobacter baumannii was $0 \%$ to colistin.

The bacterial resistance is classified by the number of antibiotics that the bacteria resist. If the bacteria are not susceptible to at least one agent in three or more antibiotic classes it is classified as a multidrug-resistant, if the bacteria are not susceptible to at least one agent in all but two or fewer antibiotic classes it is classified as extensively drug-resistant and if the bacteria are not susceptible to all agents it is classified as pandrugresistant. ${ }^{16}$ In 2018, regarding gram negative only Acinetobacter baumannii was multidrug resistant. In 2019, Klebsiella pneumonia was multidrug resistant organism in 2019 and Acinetobacter baumannii was extensively drug-resistant organism. Ahmed et al. reported that in a public hospital in Alkharj, Klebsiella pneumonia was a multidrug resistant organism and Acinetobacter baumannii bacteria were extensively drug resistance organism. ${ }^{15}$ Ruiz et al. stated that in critically ill patients, $17.9 \%$ of the patients were positive for multidrug resistant Klebsiella pneumonia. ${ }^{17}$

The present study showed that Acinetobacter baumannii was extensively drug-resistant organism. Acinetobacter baumannii is one of the 6 ESCAPE organisms that also include Enterococcus faecium, Staphylococcus aureus, Clostridioides (formerly Clostridium) difficile, Pseudomonas aeruginosa and Enterobacteriaceae. This group include clinically important and predominantly health care-associated bacteria that have the potential for significant antimicrobial resistance. ${ }^{18,19}$ Moreover, Carbapenem-resistant Acinetobacter baumannii is nowadays one of the critical-priority microbes on the World Health Organization priority list of antibiotic-resistant bacteria for effective drug development. ${ }^{20}$ The prevalence of Carbapenem resistant Acinetobacter baumannii in Saudi Arabia have also increased intensely over the years. A recent study from Riyadh reported that the susceptibilities of Acinetobacter baumannii to meropenem and imipenem were changed from a range of $64-81.2 \%$ in 2006 to the susceptibility ranged between $8.3-11 \%$ in $2012 .{ }^{21}$ The result of the present study showed that colistin is an appropriate treatment for Acinetobacter baumannii infections because the resistance rate of Acinetobacter baumannii to colistin was only $1 \%$ in 2018 and $0 \%$ in 2019. Polymyxin B and colistin (polymyxin E) are the most commonly used agents for Acinetobacter isolates that are resistant to the first-line agents. Polymyxins, such as colistin, generally have in vitro activity against Acinetobacter. ${ }^{22,23}$ Nowadays, resistance to polymyxins has been observed. ${ }^{24,25}$ In one surveillance report, the resistance rate of Acinetobacter baumannii to colistin was 2.7 percent of clinical isolates in Europe and 1.7 percent in North and Latin America. ${ }^{24}$ Zilberberg et al. reported that colistin resistance in Acinetobacter baumannii isolates during 2009 to 2012 in the United States was 6.9 percent. ${ }^{26}$

Regarding gram positive bacteria in 2018, the resistance rate generally was low. Coagulase -ve Staphylococcus was highly resistant to ciprofloxacin (70\%) and to oxacillin (60\%). Group B Streptococcus was highly resistant to tetracycline (70\%). Regarding gram positive bacteria in 2019, Staphylococcus aureus was resistant only to oxacillin (53\%) and nitrofurantoin (50\%). Coagulase -ve Staphylococcus was resistant to erythromycin $(84 \%)$, oxacillin (81\%) and clindamycin $(53 \%)$. So, there were no multidrug resistant gram-positive bacteria in 2018 and in 2019, Coagulase -ve Staphylococcus was a multidrug resistant organism in
2019. May et al. study demonstrated that the antibiotic resistance in Coagulase -ve Staphylococcus pathogens has increased significantly over the previous years. ${ }^{27}$ Marincola et al. stated that the infections that are associated with Coagulase -ve Staphylococcus from healthcare environments are typically characterized by pronounced antimicrobial resistance including both methicillin- and multidrug-resistant isolates. ${ }^{28}$

Centers for Disease Control and Prevention listed some bacterial resistant threats into three categories which are urgent threats, serious threats and concerning threats. Each category includes several resistant bacteria. ${ }^{29}$ In the present study, there was one urgent threat that was Carbapenem-resistant Acinetobacter. There was one serious threat that was Ciprofloxacin-resistant Salmonella. There was no concerning threats in the present study because concerning threats include 2 subcategories, Erythromycin-Resistant Group A Streptococcus that was not present in this study and Clindamycin-resistant Group B Streptococcus that was not tested in the present study.

In general, the resistance rate was low for most bacteria except for Klebsiella pneumonia, Coagulase -ve Staphylococcus and Acinetobacter baumannii. If we compare the resistance rate in 2018 and 2019, we could find that the resistance rate was increased in 2019. The main change in the resistance rate was for Klebsiella pneumonia. In 2018, the resistant rate of Klebsiella pneumonia to all antibiotics except nitrofurantoin was less than $50 \%$ but in 2019, the resistance rate of Klebsiella pneumonia to 9 antibiotics was more than $50 \%$.

\section{CONCLUSION}

In the present study, there were a high resistance rate of Klebsiella pneumonia, Coagulase -ve Staphylococcus and Acinetobacter baumannii mainly and there were 2 main threats which include Carbapenemresistant Acinetobacter and Ciprofloxacin-resistant Salmonella. The causes of increasing resistance rate should be determined to decrease the occurrence of infections that are caused by multidrug resistant organisms. Moreover, it is recommended to increase the awareness of the health care professionals regarding the appropriate prescribing of antibiotics based on antimicrobial resistance rate and to increase the awareness of the public about the wise use of antibiotics.

\section{Limitations}

The main limitations in the study were that resistance of some bacteria to numerous antibiotics was not tested. For example, the resistance rate of Group B Streptococcus to clindamycin was not tested in the present study and Clindamycin-resistant Group B Streptococcus are considered as a concerning threat. Furthermore, there was a lack of information about the previous use of antibiotics so if this information will be available in the future, the researchers could find the relation between the bacterial resistance and antibiotics use.

\section{ACKNOWLEDGEMENT}

This Publication was supported by the Deanship of Scientific Research at Prince Sattam bin Abdulaziz University.

\section{CONFLICT OF INTEREST}

The authors declare that there is no conflict of interest.

\section{REFERENCES}

\footnotetext{
1. Nathan C, Cars O. Antibiotic resistance--problems, progress, and prospects N Engl J Med. 2014:371(19):1761-3. doi: 10.1056/NEJMp1408040, PMID 25271470.

2. Nathan C. Antibiotics at the crossroads. Nature. 2004;431(7011):899-902. doi: 10.1038/431899a, PMID 15496893.
} 
3. Levy SB. Antibiotic resistance: an ecological imbalance. Ciba Found Symp. 1997;207:1-9; discussion 9. doi: 10.1002/9780470515358.ch1, PMID 9189631.

4. Méndez-Vilas A. Microbial pathogens and strategies for combating them: science. J Technol Educ. 2013;1:291-6.

5. Popeda M, Płuciennik E, Bednarek AK. Proteins in cancer multidrug resistance. Postepy Hig Med Dosw. 2014;68:616-32. doi: 10.5604/17322693.1103268.

6. Van Duin D, Paterson DL. Multidrug-resistant bacteria in the community: trends and lessons learned. Infect Dis Clin North Am. 2016;30(2):377-90. doi: 10.1016/j. idc.2016.02.004, PMID 27208764

7. Tanwar J, Das S, Fatima Z, Hameed S. Multidrug resistance: an emerging crisis. Interdiscip Perspect Infect Dis. 2014;2014:541340. doi: 10.1155/2014/541340.

8. Infectious Diseases Society of America (IDSA), Spellberg B, Blaser M, Guidos RJ, Boucher HW, Bradley JS, et al. Combating antimicrobial resistance: policy recommendations to save lives. Clin Infect Dis. 2011;52;Suppl 5:S397-428. doi: 10.1093/cid/cir153, PMID 21474585.

9. Vardakas KZ, Rafailidis PI, Konstantelias AA, Falagas ME. Predictors of mortality in patients with infections due to multi-drug resistant Gram negative bacteria: the study, the patient, the bug or the drug? J Infect. 2013;66(5):401-14. doi: 10.1016/j.jinf.2012.10.028, PMID 23142195

10. Bodi M, Ardanuy C, Rello J. Impact of Gram-positive resistance on outcome of nosocomial pneumonia. Crit Care Med. 2001;29(4);Suppl:N82-6. doi: 10.1097/00003246-200104001-00005, PMID 11292880

11. Nikaido H. Multidrug resistance in bacteria. Annu Rev Biochem. 2009;78:119-46. doi: 10.1146/annurev.biochem.78.082907.145923, PMID 19231985.

12. Who. Int [homepage on the Internet]. Antimicrobial resistance: global report on surveillance. updated 2014 June.

13. Magiorakos AP, Srinivasan A, Carey RB, Carmeli Y, Falagas ME, Giske CG, et al. Multidrug-resistant, extensively drug-resistant and pandrug-resistant bacteria: an international expert proposal for interim standard definitions for acquired resistance. Clin Microbiol Infect. 2012;18(3):268-81. doi: 10.1111/j.14690691.2011.03570.x, PMID 21793988.

14. Bono L, Li Cavoli G, Verde MS, Sodano C, Tortorici C, Ferrantelli A, Li Cavoli TV, Rotolo U. Prevalence of bacterial pathogens and their emerging resistance patterns in patients with renal diseases. Dial Trasplant. 2015;36(2):78-82. doi: 10.1016/j.dialis.2015.02.006

15. Ahmed NJ, Haseeb A, Hassali MA, Elsaid EE, Khan AH. Antimicrobial resistance of ESKAPE pathogens in a public hospital in AlKharj. Lat Am J Pharm. 2021:40(3):442-50

16. Uptodate. Acinetobacter infection: treatment and prevention [online]; 2021 [cited Sep 01 2021]. Available from: https://www.uptodate.com/contents/acinetobacter-infection-treatment-and-prevention/.

17. Ruiz A J, Gordon M, Villarreal E, Frasquet J, Sánchez MÁ, Martín M, Castellanos Á, Ramirez P. Influence of antibiotic pressure on multi-drug resistant Klebsiella pneumoniae colonisation in critically ill patients. Antimicrob Resist Infect Control, $2019 ; 8(1): 1-7$.
18. Rice LB. Federal funding for the study of antimicrobial resistance in nosocomial pathogens: no ESKAPE. J Infect Dis. 2008;197(8):1079-81. doi: 10.1086/533452 PMID 18419525

19. De Rosa FG, Corcione S, Pagani N, Di Perri G. From ESKAPE to Escape, from KPC to CCC. Clin Infect Dis. 2015:60(8):1289-90. doi: 10.1093/cid/ciu1170, PMID 25537879.

20. Tacconelli E, Carrara E, Savoldi A, Harbarth S, Mendelson M, Monnet DL, et al. Discovery, research, and development of new antibiotics: the WHO priority list of antibiotic-resistant bacteria and tuberculosis. Lancet Infect Dis. 2018;18(3):318-27. doi: 10.1016/S1473-3099(17)30753-3, PMID 29276051.

21. Al-Obeid S, Jabri L, Al-Agamy M, Al-Omari A, Shibl A. Epidemiology of extensive drug resistant Acinetobacter baumannii (XDRAB) at Security Forces Hospital (SFH) in Kingdom of Saudi Arabia (KSA). J Chemother. 2015;27(3):156-62. doi: 10.1179/1973947815Y.0000000019, PMID 25867622.

22. Linden PK, Paterson DL. Parenteral and inhaled colistin for treatment of ventilator-associated pneumonia. Clin Infect Dis. 2006;43;Suppl 2:S89-94. doi: 10.1086/504485, PMID 16894521.

23. Horton J, Pankey GA. Polymyxin B, colistin, and sodium colistimethate. Med Clin North Am. 1982;66(1):135-42. doi: 10.1016/s0025-7125(16)31447-x, PMID 6278236 .

24. Halstead DC, Abid J, Dowzicky MJ. Antimicrobial susceptibility among Acinetobacter calcoaceticus-baumannii complex and Enterobacteriaceae collected as part of the tigecycline Evaluation and Surveillance Trial. J Infect. 2007:55(1):49-57. doi: 10.1016/j.jinf.2006.11.018, PMID 17250897.

25. Qureshi ZA, Hittle LE, O'Hara JA, Rivera JI, Syed A, Shields RK, Pasculle AW Ernst RK, Doi Y. Colistin-resistant Acinetobacter baumannii: beyond carbapenem resistance. Clin Infect Dis. 2015;60(9):1295-303. doi: 10.1093/cid/civ048, PMID 25632010 .

26. Zilberberg MD, Kollef MH, Shorr AF. Secular trends in Acinetobacter baumannii resistance in respiratory and blood stream specimens in the United States, 2003-2012: A survey study. J Hosp Med. 2016;11(1):21-6. doi: 10.1002/ jhm.2477, PMID 26353076.

27. May L, Klein EY, Rothman RE, Laxminarayan R. Trends in antibiotic resistance in coagulase-negative staphylococci in the United States, 1999-2012. Antimicrob Agents Chemother. 2014;58(3):1404-9. doi: 10.1128/AAC.01908-13, PMID 24342646.

28. Marincola G, Liong O, Schoen C, Abouelfetouh A, Hamdy A, Wencker FDR Marciniak T, Becker K, Köck R, Ziebuhr W. Antimicrobial resistance profiles of coagulase-negative staphylococci in community-based healthy individuals in Germany. Front Public Health. 2021;9:684456. doi: 10.3389/fpubh.2021.684456, PMID 34222184.

29. CDC. CDC's antibiotic resistance threats in the United States [online]; 2019 [cited Sep 01 2021]. Available from: https://www.cdc.gov/drugresistance/ biggest-threats.html/.

Article History: Received: 04-09-2021; Revised: 29-09-2021; Accepted: 21-10-2021.

Cite this article: Ahmed NJ, Haseeb A, Mahmoud S, Khan AH. Emergence of High Drug Resistant Bacterial Isolates in Al-Kharj. J Young Pharm. 2021;13(4):386-91. 